\title{
Grey matter pathology in multiple sclerosis
}

\author{
Jeroen J G Geurts, Frederik Barkhof
}

\begin{abstract}
Multiple sclerosis (MS) has been classically regarded as a white matter disease. However, recent histopathological studies have convincingly shown that grey matter regions are also heavily affected. Grey matter damage starts early in the disease and substantially affects clinico-cognitive functioning. Detection of cortical grey matter lesions by use of standard MRI techniques has proved challenging, and more advanced techniques are needed. At present, the causes of grey matter damage are unclear. We review several exciting new hypotheses on grey matter pathogenesis, including meningeal inflammation as a cause of subpial cortical damage, but also selective vulnerability of neuronal subpopulations, growth factor dysregulation, glutamate excitotoxicity, mitochondrial abnormalities, and the "use-itand-lose-it" principle. These hypotheses remain to be validated over the coming years, and could substantially affect our current views on MS pathogenesis.
\end{abstract}

\section{Introduction}

Multiple sclerosis (MS) is an inflammatory demyelinating disease of the CNS that is prevalent among young adults and usually leads to chronic disability. Although MS has been classically thought of as a typical white matter disorder, the involvement of grey matter regions in the demyelinating process was acknowledged in early pathology studies..$^{1-5}$ However, mainly owing to poor visualisation of demyelination within grey matter areas with conventional histological staining methods (figure 1), ${ }^{6}$ the issue had long been disregarded. Since the introduction of myelin immunohistochemistry, the subject of demyelination in grey matter regions in MS has received new impetus, ${ }^{7-9}$ and demyelination of axons in the cortex has been reported to be extensive and at least partly independent of the presence of white matter lesions. ${ }^{6,7,10}$

Clinically, demyelination of axons in the cortex and other grey matter regions is of importance, because focal demyelination in the white matter, visible on MRI, cannot explain the full extent of clinical, including cognitive, deficits in patients with MS. ${ }^{11}$ White matter lesion load, (juxta)cortical lesions, and brain atrophy, as determined by MRI, have all been used to explain part of the physical disability, epilepsy, depression, and cognitive impairment seen in MS. ${ }^{12-18}$ However, specific cognitive deficits, such as memory impairment, attention deficits, and reduced mental processing, which can be found in $45-65 \%$ of patients with $\mathrm{MS},{ }^{19,20}$ might be better explained by pathological processes (ie, demyelination and possible resultant damage to the neurons) in the grey matter. This was illustrated by the fact that MS patients with cognitive decline have more cortical damage than patients who are cognitively preserved. ${ }^{21,22}$ Furthermore, a rarely occurring cortical variant of MS was defined, with patients showing predominant or exclusive cognitive decline starting from disease onset. ${ }^{23,24}$

Since the recent revival of research on grey matter abnormalities in MS, much effort has been put into describing and classifying pathology within grey matter areas, as well as in imaging grey matter lesions and relating these lesions to clinical disability. However, the causes of damage in grey matter regions, which mainly consists of demyelination of axons, but also involves neuroaxonal degeneration in the grey matter, remain unknown. Recently, several new hypotheses have been put forward that have explored possible pathogenic processes leading to grey matter damage. These processes could be either primary (arising within grey matter regions) or secondary (pathological changes in grey matter regions that result from continuing damage in the cerebral white matter) and might be intricately connected with each other. The aim of our Review is to discuss these new ideas, put them into perspective, relate them to white matter pathology and clinico-cognitive impairment, and provide a comprehensive overview of grey matter pathology research in MS.

\section{Grey matter pathology in MS}

A classification system for cortical grey matter lesions was proposed, which distinguishes type I mixed white matter-grey matter lesions from types II, III, and IV purely intracortical lesions (figure 2). ${ }^{7,825}$ Type III, or subpial, cortical lesions are most common and can extend over several gyri to involve nearly all cortical areas sampled at autopsy. ${ }^{6,7}$ In addition to the neocortex, demyelination can be found in other grey matter areas, such as the thalamus, basal ganglia, hypothalamus, hippocampus, cerebellum, and spinal cord (figure 2)..$^{10,25-28}$ Extensive demyelination in the cortex (figures 1 and 3 ) is associated with a more progressive phase of the disease, ${ }^{10}$ and (juxta)cortical, hypothalamic, and hippocampal lesions have been shown to correlate with the degree of clinical disability. ${ }^{12,14,25,29,30}$ The pathology of grey matter lesions differs from that of white matter lesions in that significant lymphocyte infiltration, complement deposition, and blood-brain barrier (BBB) disruption have so far not been detected in grey matter lesions, whereas white matter lesions are usually inflammatory., ${ }^{8,31,32}$ However, axonal transection, in addition to neuronal, glial, and synaptic loss, can be found to some extent in cortical grey matter lesions, ${ }^{9,33}$ which seems important in understanding the consistent finding of atrophy and cortical thinning in MS, as measured by MRI..$^{34-39}$ In a histopathological study, Wegner and colleagues ${ }^{33}$ described a $10 \%$ overall cortical thickness reduction in their MS cases. Whether this
Lancet Neurol 2008; 7: 841-51 Department of Radiology (J) G Geurts PhD, F Barkhof MD) and Department of Pathology (J) G Geurts), VU University Medical Centre, Amsterdam, Netherlands

Correspondence to: JJ G Geurts, VU University Medical Centre, Department of Radiology, Room PK 0X-112, De Boelelaan 1117, $1081 \mathrm{HV}$ Amsterdam, Netherlands j.geurts@vumc.nl 


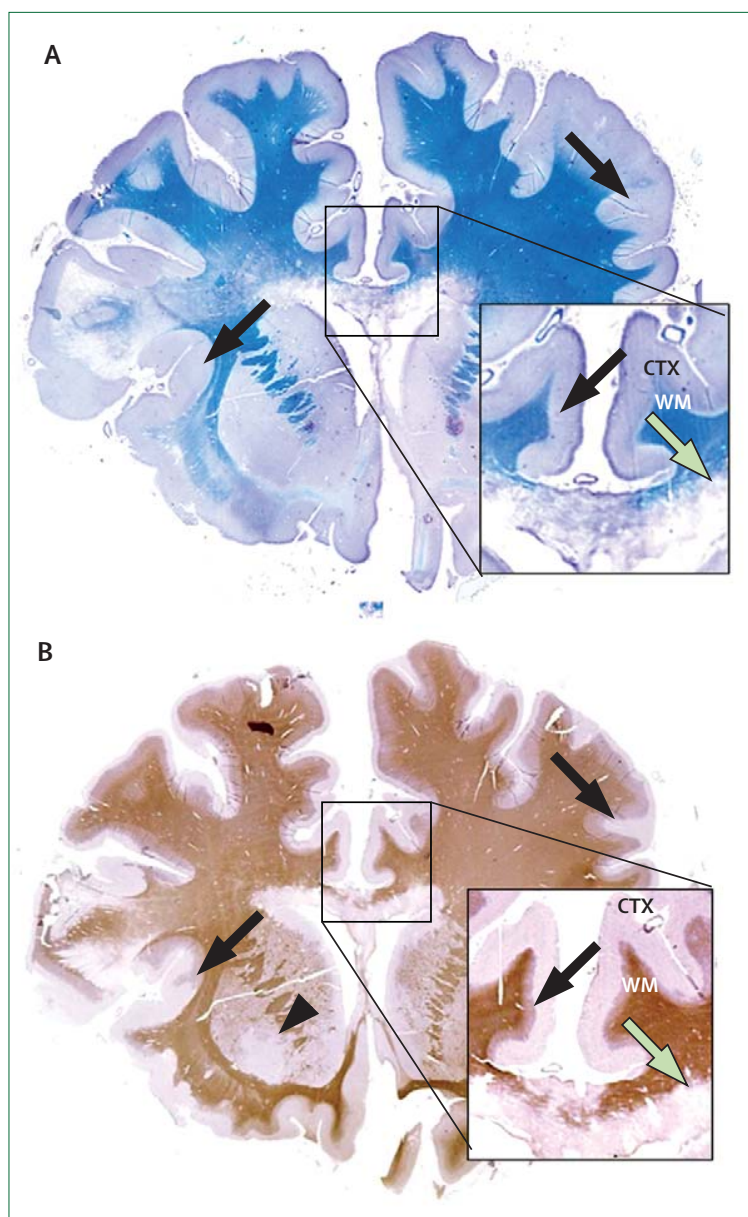

Figure 1: Adjacent paraffin sections from an MS patient with general cortical subpial demyelination

Sections have been (A) histochemically stained for myelin by use of the Luxol fast-blue technique and (B) immunohistochemically stained for proteolipid protein (PLP). (A) Demyelination of periventricular white matter (WM), including the corpus callosum, is readily detectable (green arrow); cortical myelin is largely unstained (black arrows). (B) Periventricular lesions are also well delineated by PLP immunohistochemistry (green arrow). In the cerebral cortex (CTX), all areas have a superficial subpial loss of myelin (black arrows); an area of myelin loss is also detected in the putamen (arrowhead). At higher

magnification, a sharply defined cortical lesion border (cingulate gyrus) is visible by PLP immunohistochemistry ( $B$; inset, black arrow); the border is not detectable by Luxol fast-blue staining in an adjacent section ( $A$; inset, black arrow). Reproduced from Bö et al, ${ }^{6}$ with permission from the American Medical Association.

global thinning is a secondary result of accumulating focal demyelination in cortical areas, or whether substantial neuroaxonal loss also occurs outside focal cortical lesions, is unclear. The pathological substrate that underlies cortical volume reduction early in the disease is also unclear ${ }^{35,38}$ because demyelination of axons in the cortex should still be relatively limited at that stage. ${ }^{10}$

Cortical thinning on MRI was found to be most prominent in frontal and temporal areas,,$^{34}$ which is in agreement with early histopathological findings on the topography of demyelination in the cortex..$^{40,4}$ Those and other (MRI) studies provided further evidence that
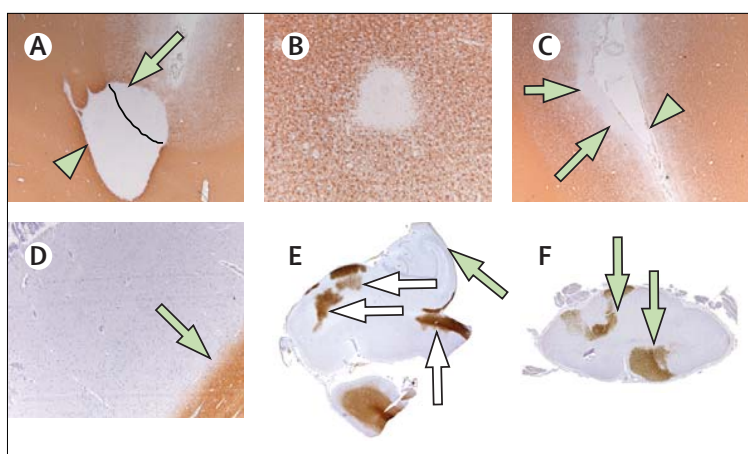

Figure 2: Different presentations of grey matter lesions (myelin immunohistochemistry, proteolipid protein)

Panels A-D show four types of cortical grey matter lesions. ${ }^{8}$ (A) Type I or mixed white matter-grey matter lesion: the black line indicates the border between the cortex and subcortical white matter, and the grey matter and white matter parts of the lesion are indicated by the arrow and arrowhead, respectively (original magnification 2.5x). (B) Type II intracortical lesion surrounding a blood vessel (original magnification $5 \times$ ). (C) Type III subpial cortical lesion: arrows indicate the outline of a type III lesion, with demyelination restricted to the upper layers of the cortex. Adjacent normal cortex is indicated by the arrowhead (original magnification 2.5x). (D) Type IV lesion, which spans the entire cortical ribbon (original magnification $5 \times$ ): arrow indicates the grey matter-white matter border. (E) Hippocampal lesion (original magnification $3 \times$ ). These lesions are frequent and extensive, and are often mixed (involving both the hippocampus and the perihippocampal white matter). The green arrow indicates the fully demyelinated hippocampal subfields, and the white arrows point to areas left intact with myelin in the perihippocampal grey matter and white matter. (F) Spinal cord lesion (original magnification $3 \times$ ); only some small areas containing myelin are remaining (arrows). (E, F) Images obtained with a Zeiss Mirax Scan, courtesy of Jeroen Beliën (Department of Pathology, VU University Medical Centre, Amsterdam, Netherlands). (E) Reproduced from Geurts et al, with permission from Lippincott Williams and Wilkins.

(cortical) grey matter pathological changes importantly contribute to clinical disease severity. ${ }^{22,3436,42,43}$ However, direct effects of focal cortical grey matter lesions on clinical measures, including cognition, have not yet been reliably established. Study of the relation between the occurrence of cortical lesions and clinico-cognitive functioning is challenging, because visualisation of demyelination in cortical grey matter regions in vivo with MRI has hitherto proved to be difficult; in particular, subpial (type III) cortical lesions could not be detected..$^{44,45}$

\section{Imaging of grey matter lesions Advanced MRI techniques}

In a search for new, more advanced imaging techniques to improve cortical lesion visualisation, a multi-slab three-dimensional (3D) double inversion recovery (DIR) technique was developed and applied to an MS population, which enabled a five times increase in cortical lesions detected compared with more standard MRI techniques (figure 4). ${ }^{46}$ This 3D-DIR was further developed into a single-slab, isotropic version, allowing for a substantially reduced acquisition time, and a clear reduction of slice-profile and flow artifacts (figure 5). ${ }^{47}$ Furthermore, a T1-weighted 3D spoiled gradient-recalled echo (SPGR) sequence was recently reported to be useful in the detection of cortical lesions. ${ }^{48}$ 
As judged from the number of cortical plaques reported in post-mortem studies, ${ }^{44}$ not all cortical lesions can be detected by (3D)-DIR or T1-based MRI sequences. It might well be that only new, possibly slightly more inflammatory (or oedematous), lesions can be observed, ${ }^{29}$ whereas the more chronic cortical lesions remain invisible on in vivo MRI. However, inflammation might not be integral to demyelination in the cortex, except in the type I mixed white matter-grey matter lesions. ${ }^{8}$ Of note, an inescapable selection bias exists when comparing post-mortem tissue to the situation in vivo: cases examined at autopsy usually had chronic MS before death, and are thus likely to have built up more (chronic) grey matter damage than cases examined in life. ${ }^{10}$ Application of 3D-DIR, probably the most effective technique for the detection of cortical lesions in vivo, to the post-mortem situation should be actively pursued, because it might reveal exactly how many cortical (and other) grey matter lesions are missed by MRI, and which specific pathological hallmark (or lack thereof) is responsible for cortical lesions being visible or invisible on MRI. However, whether newer MRI methods will enable detection of all cortical lesions, and more specifically of all subpial cortical lesions in the future, cannot be ensured. High-field MRI, which uses substantially prolonged scanning times and high signalto-noise ratio, has not led to convincingly improved cortical lesion visualisation to date. ${ }^{45}$

\section{Imaging of normal-appearing grey matter}

Because of the abovementioned difficulties concerned with the imaging of cortical lesions in vivo, several MRI studies have reverted to measuring abnormalities in normalappearing grey matter (NAGM; ie, grey matter that looks normal on conventional MRI, but is probably not histopathologically normal) by use of various techniques, including magnetisation transfer ratio (MTR) imaging, ${ }^{49-51}$ T1-relaxation time measurements, ${ }^{42,52}$ diffusion tensor imaging (DTI), ${ }^{53,54}$ and proton magnetic resonance spectroscopy (MRS). ${ }^{55-57}$ However, the pathological substrate that is responsible for these NAGM changes is unclear. As mentioned, apart from global cortical thinning, ${ }^{33}$ little is known about pathological findings outside focal cortical lesions. Abnormalities (transected neurites) outside focal lesions were shown to be much more subtle than abnormalities within cortical lesions.' Therefore, the abnormal NAGM MRI findings are more likely to be influenced by the cortical lesions that remain largely undetected on MRI..$^{58}$ A beneficial application of DIR, SPGR imaging, or other MRI techniques more sensitive to cortical lesions, lies in segmenting out grey matter lesions to achieve a more accurate and reliable investigation of "real" NAGM (ie, the non-lesional grey matter).

In terms of clinical correlations, the study of quantitative MRI and atrophy measures of NAGM has proved worthwhile. $^{59}$ Cortical grey matter atrophy studies (essentially a measure of sulcal widening) showed
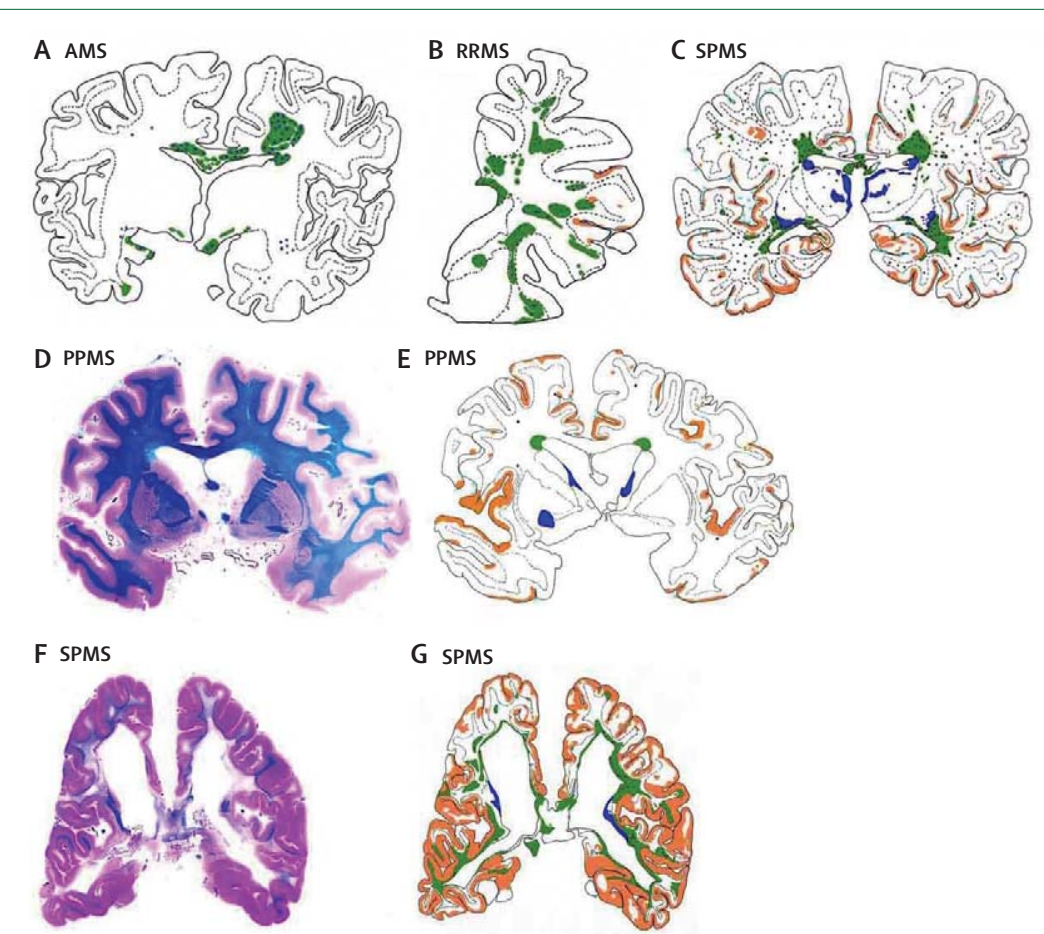

G SPMS
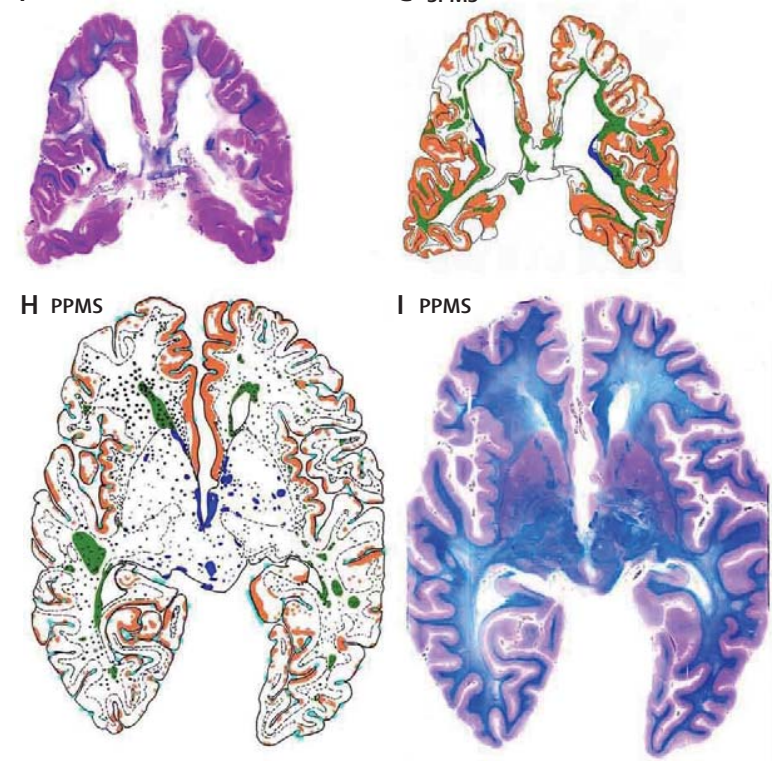

Figure 3: Focal inflammatory demyelinated plaques in white matter dominate the lesions seen in acute MS (AMS) and relapsing-remitting MS (RRMS), whereas cortical demyelination and diffuse white matter inflammation are characteristic of primary progressive MS (PPMS) and secondary progressive MS (SPMS) $(A-C, E, G, H)$ Schematic lesion maps of MS brains and $(D, F, I)$ corresponding sections stained with Luxol fast-blue stain. Green areas show focal demyelinated plaques in white matter; orange areas show cortical demyelination; blue areas show demyelinated lesions in deep grey matter; dark blue dots indicate inflammatory infiltrates in the brain; and light blue dots indicate inflammatory infiltrates in the meninges. (A) Acute MS in a 35-year-old man with disease of 1.5 months' duration. (B) Relapsing-remitting MS in a 57-year-old woman with disease of 13 years' duration. (C) Secondary progressive MS in a 43-year-old man with disease of 16 years' duration. (D, E) Primary progressive MS with severe demyelination in the cortex, but only minor disease involvement of white matter. $(F, G)$ Secondary progressive MS in a 46-year-old woman with disease of 16 years' duration, showing extensive damage in cortex and white matter. $(\mathrm{H}, \mathrm{I})$ Primary progressive MS in a 55-year-old woman with disease of 5 years' duration, showing diffuse white matter abnormalities; only the subcortical myelin is intact, and only a few focal demyelinated plaques are visible. Reproduced from Kutzelnigg et $\mathrm{al}_{1}{ }^{10}$ with permission from Oxford University Press.

significant correlations with clinical disability, ${ }^{35,60-62}$ as well as cortical thickness measurements, ${ }^{34,36,43}$ which reported specific focal cortical thinning in frontal, temporal, parietal, precentral, and anterior cingulate cortex, consistent with histopathological reports on the preferential distribution of demyelination in the cortex.? 


\section{Review}
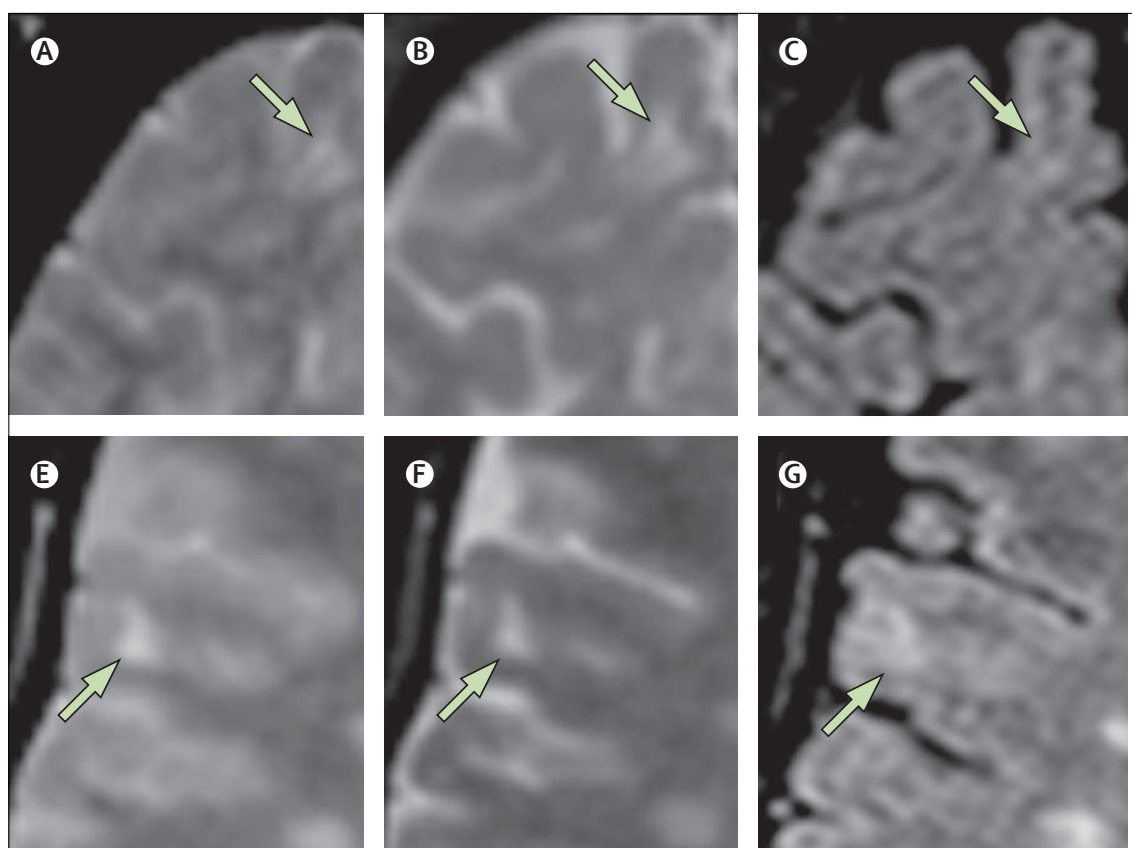

IW

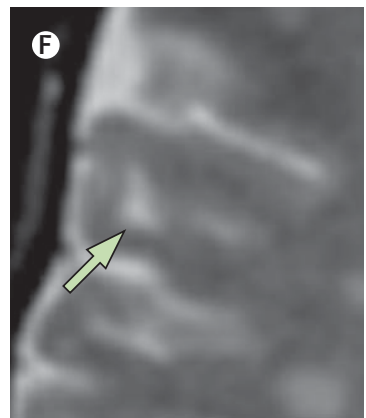

T2

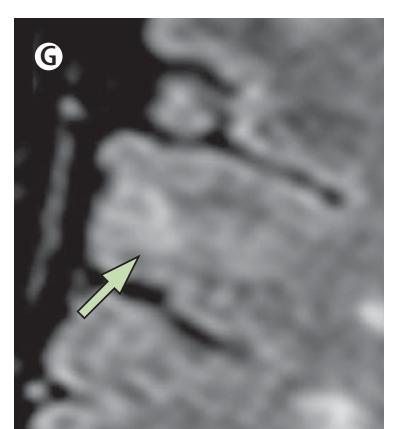

FLAIR
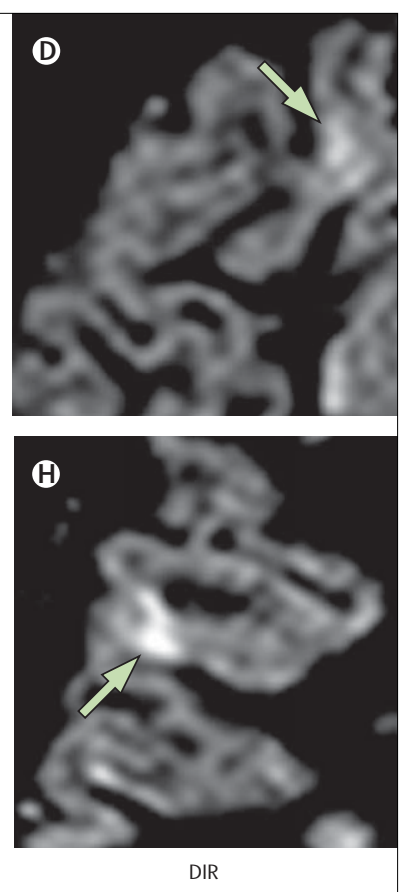

DIR

Figure 4: Transverse intermediate-weighted, T2-weighted, 3D-FLAIR, and 3D-DIR images of intracortical lesions

Panels A-D show the lesion (arrow) in cortical grey matter, with a possible juxtacortical component; the intracortical lesion is particularly poorly visible on intermediate-weighted (IW) and T2-weighted images, and on the fluid-attenuated inversion recovery (FLAIR) image, whereas it is depicted clearly on the double inversion recovery (DIR) image. Panels E-H (different patient): DIR image shows very good delineation of the intracortical lesion (arrow), which could be mistaken for a juxtacortical lesion or a partial volume artefact on the T2-weighted image and might even be missed on the FLAIR image. No contrast agent was used. Reproduced from Geurts et al ${ }^{46}$ with permission from the Radiological Society of North America.
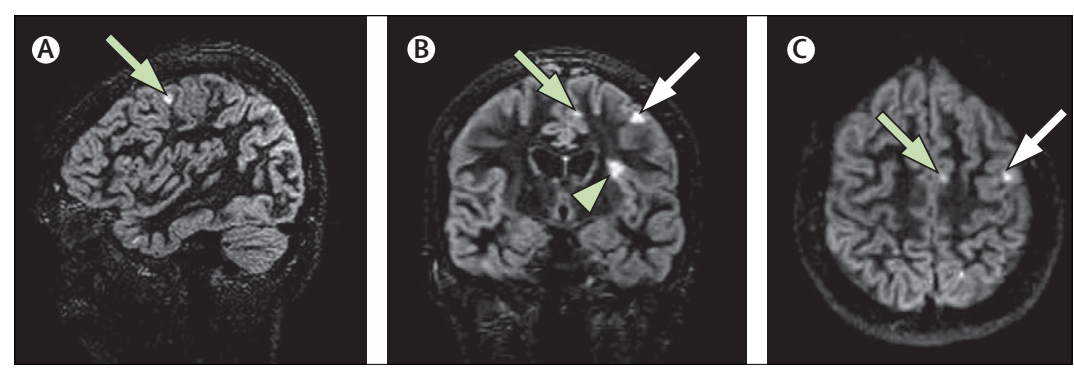

Figure 5: Multiplanar representation of single-slab 3D-DIR images of a patient with MS

Compared with the multi-slab 3D-DIR (see figure 4), this newly developed single-slab, isotropic sequence has the advantage of shorter acquisition times, improved signal-to-noise properties and easy reformatting to allow the best visualisation of (small) grey matter lesions. (A) Sagittal view of a juxtacortical lesion (arrow) in the frontal vertex. (B) Coronal orientation: same wedge-shaped juxtacortical lesion (white arrow), as well as a mixed grey matter-white matter (type I) lesion (arrowhead) near the frontal operculum, and a smaller juxtacortical lesion frontomedially (green arrow). (C) Same two juxtacortical lesions as shown in (A) and (B), in the axial orientation.

Several studies showed that quantitative MRI measures, such as MTR, ${ }^{63-66}$ DTI, ${ }^{53,54}$ and T1-relaxation times, ${ }^{42,52}$ of the grey matter were related to, or even predictive of, disability in MS. Furthermore, changes in several brain metabolites, as detected by MRS and whole-brain MRS imaging, could be related to disability..$^{55,67-69}$ Not only cortical, but also deep grey matter structures were found to be abnormal in terms of metabolite concentrations, ${ }^{56,70}$ which could be partly confirmed histopathologically. ${ }^{70}$ Furthermore, T2 hypointensities possibly resulting from increased iron deposition in the basal ganglia were shown to be related to brain atrophy and clinical disability. ${ }^{71}$ As for MRS data, a recent meta-analysis found that metabolite concentrations could vary substantially between studies ${ }^{72}$ and reproducibility and accuracy of the MRS experiment are strongly dependent on the brain region and the metabolite assessed. ${ }^{3}$

Currently, some controversy exists about the relation between NAGM and white matter abnormalities. Although it seems attractive to assume that grey matter damage arises secondarily to accumulating white matter damage (both focal and in the normal-appearing white matter), several MRI and histopathology studies seem to indicate that grey matter and white matter abnormalities occur largely independently, ${ }^{6,10,21,35}$ or at least evolve in different time-frames. ${ }^{10}$

\section{Spinal cord imaging}

MS lesions are also frequently found in the spinal cord of patients with MS, and although spinal lesions have been observed to affect both grey matter and white matter structures, a recent histopathology study showed that the proportion of demyelination of grey matter regions is generally larger in the MS cord. ${ }^{28}$ Conventional MRI of the spinal cord in patients with MS was shown to be feasible and highly disease specific, ${ }^{74}$ which led to a more prominent role for spinal MS lesions in the diagnostic criteria. ${ }^{75,76}$ Both focal and diffuse lesions can be seen on MRI of the spinal cord, the latter being 
associated with a more progressive clinical course and greater disability."

Mainly due to technical difficulties, progress in the more advanced (non-conventional) MRI assessment of the spinal cord has been slow. However, recent quantitative MRI studies of the MS cervical spinal cord consistently reported clinically relevant reductions in total $\mathrm{N}$-acetylaspartate, decreased fractional anisotropy, increased mean diffusivity, increased cord atrophy, and functional signal changes when compared with healthy controls..$^{78-82}$

Functional MRI of (normal-appearing) grey matter in MS Over the past few years, functional MRI has been successfully applied to show differences in brain activation between patients with MS and healthy individuals. Functional cortical changes were found in response to motor tasks and as a result of accumulating white matter lesions,$^{83}$ and were strongly correlated with axonal damage as measured by $\mathrm{MRS},{ }^{84}$ and with damage in normal-appearing brain tissue as measured by MTR imaging and DTI. ${ }^{85,86}$ Functional cortical changes were also specifically found in relation to cognitive processing, ${ }^{87-89}$ even at the earliest stage of disease..$^{90}$

The existence of a discrepancy between structural and functional deficits in MS is well known. ${ }^{11}$ Therefore, several studies have used functional MRI to focus on the issue of plasticity within the brain and spinal cord, and have subsequently shown that, after structural damage, functional adaptation and reorganisation might take place to a certain extent, ${ }^{82,86,91-93}$ depending on disease stage. ${ }^{91}$ These findings can now be uniquely combined with novel techniques such as diffusion tractography ${ }^{81,94}$ and tract-based spatial statistics ${ }^{95}$ to study the direct effects of structural connectivity changes on functional connectivity. A recent example of such an innovative combination of techniques is provided by Rocca and colleagues. $^{92}$ These investigators showed that patients with MS had significantly more activation in several brain areas related to motor function, in addition to increased functional connectivity between these areas, which could be directly related to structural tissue damage, as assessed by DTI metrics of the associated white matter tracts. Similarly, the concept of Wallerian degeneration (ie, anterograde axonal degeneration after transection of axons in the white matter), ${ }^{96,97}$ which is an interesting pathological concept but remains elusive in terms of both microscopic and macroscopic assessment, could thus be studied in more detail.

By use of ${ }^{18} \mathrm{~F}$-fluorodeoxyglucose PET imaging, reduced rest metabolism was found in the thalamus, cerebellum, hippocampus, and dorsolateral prefrontal, orbitofrontal, and occipital cortex of patients with MS. ${ }^{98-100}$ An especially interesting and recent development in PET is microglial imaging. ${ }^{101}$ This should be relevant in terms of detecting subtle abnormalities in (cortical) grey matter and normalappearing white matter, ${ }^{10}$ and possibly even in the in vivo
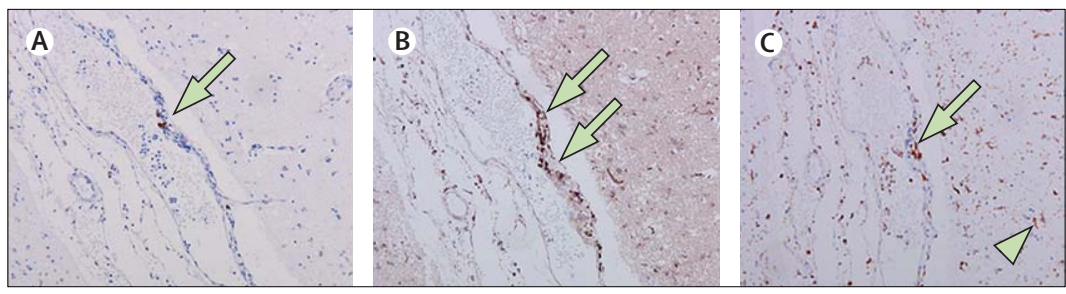

Figure 6: Meningeal inflammation in MS

(A) Immunohistochemical stain for CD20, with a few immunopositive B cells in the sulcal leptomeninges (arrow). (B) CD3 stain, showing many T cells in the meninges (arrows). (C) CD68-positive macrophages in the meninges (arrow) and reactive microglia in adjacent cortical grey matter (arrowhead). Original magnifications 10x. Unpublished data, courtesy of E-J Kooi (Department of Neuropathology, VU University Medical Centre, Amsterdam, Netherlands)

classification of grey matter (and white matter) lesion subtypes or stages. ${ }^{8}$ Finally, new insights into the functional changes of the MS brain have come from perfusion MRI, which indicates that reduced cerebral blood flow in the thalamus, putamen, and caudate nuclei of patients with MS could be related to neuropsychological impairment and fatigue. ${ }^{102-104}$

\section{Grey matter damage in MS disease subtypes}

In an important study by Kutzelnigg and colleagues, ${ }^{10}$ the evolution of demyelination in grey matter regions over time was illustrated by myelin stainings of acute, relapsing-remitting $(\mathrm{RR})$, secondary progressive $(\mathrm{SP})$, and primary progressive (PP) MS cases, indicating that although demyelination of axons in the grey matter is already present to some extent in RRMS and acute MS, it becomes much more prominent in the chronic disease stages. In SPMS and PPMS cases, demyelination of axons in the cortex was seen to concur with a diffuse microglial reactivity in the white matter, and with widespread meningeal (figure 6) and perivascular inflammation..$^{105,106}$ In a separate study by the same group, patients with SPMS and PPMS were also shown to exhibit extensive demyelination in the cerebellum. ${ }^{107}$ The histopathological finding of grey matter abnormalities early in the disease, with a significant increase in grey matter damage over time, was supported by MRI findings and biopsy studies. ${ }^{42,65,108-113}$

Although early damage to the cortex, or to the brain in a broader sense, might cause significantly higher disability in patients with $\mathrm{MS},{ }^{35}$ brain plasticity, and the so-called "reserve capacity" were shown to partially compensate for disease damage. Disease-type-dependent plasticity and functional reorganisation of movementassociated cortical networks have been described in patients with MS, ${ }^{86,91,93}$ and these were associated with abnormalities in normal-appearing brain tissue, independent of T2-visible focal white matter abnormalities. The extent to which functional (dys)connectivity is directly causally related to structural (dys)connectivity is currently unclear. It would be interesting for future studies to combine measures of white matter tract integrity, based on DTI and newly 


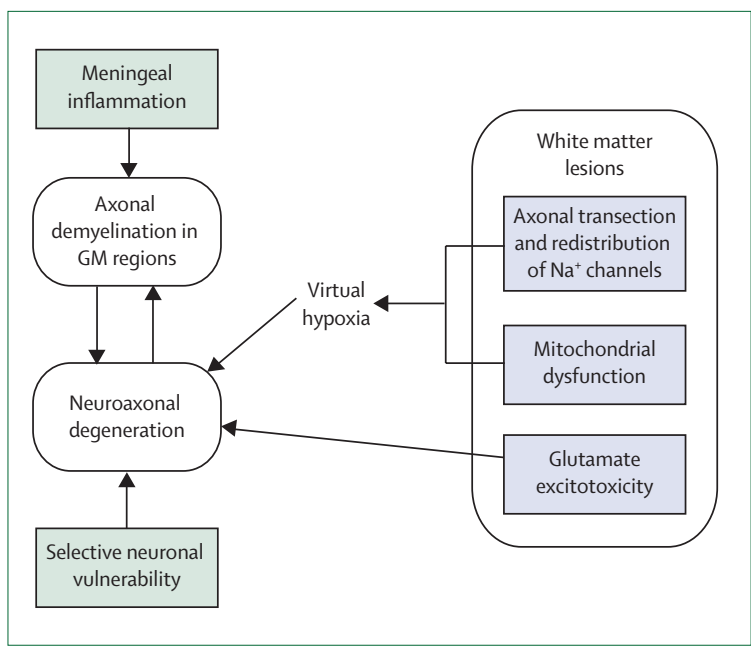

Figure 7: Schematic representation of disease processes currently believed to underlie grey matter damage in MS

Grey matter damage could occur secondarily to white matter pathology. Several secondary disease mechanisms (blue boxes) have been proposed, including the "virtual hypoxia" theory by Stys and colleagues. ${ }^{115}$ This theory postulates that axonal degeneration in the grey matter might result from inflammatory activity in white matter lesions, combined with subsequent axonal demyelination and reorganisation of sodium channels and an inadequate mitochondrial energy supply. Moreover, glutamate excitotoxicity might aggravate neuroaxonal damage in the grey matter..$^{116-121}$ This degeneration of axons and neurons in grey matter might in turn lead to demyelination and the lesions visible under the microscope in grey matter areas. Alternatively, pathological processes affecting grey matter areas primarily (green boxes), without intervening effects from white matter damage, might arise as a result of meningeal inflammation and the concomitant release of a myelinotoxic substance, ultimately leading to subpial cortical demyelination. ${ }^{105}$ This might cause demyelination of axons in the grey matter, which in turn could result in neuroaxonal degeneration.

Furthermore, primary cortical degeneration might occur in specific predilection sites, as is seen in dementias; for example, animal studies have shown that the cholinergic system is specifically involved in the disease. Led by functional and cortical thinning studies, consistently active brain areas (eg, default-mode networks) could be hypothesised to be specifically subject to neurodegeneration (the "use-it-and-lose-it" principle). Of note, the primary and secondary disease processes proposed do not imply sequentiality, but are instead likely to develop largely independently, with cumulative effects on grey matter damage in MS.

developed statistical modelling methods, ${ }^{95}$ with functional connectivity, as measured with functional MRI, for example. ${ }^{114}$ Thus, clinico-cognitive functioning might be approached from the point of view of residual capacity (ie, the remaining, unaffected function of the brain), which in turn might be advantageous in terms of rehabilitation and therapeutic strategies. Moreover, in the context of plotting the time-course of the pathological processes of MS, the study of the relative contributions of white matter and grey matter damage to impaired clinicocognitive functioning in every stage of the disease remains important.

\section{Causes of grey matter damage}

As mentioned, there is little or no significant inflammation, BBB damage, or gliosis in (cortical) grey matter lesions, ${ }^{8,31,32}$ as opposed to white matter lesions, in which extensive Tcell inflammation, presence of (foamy) macrophages, BBB disruption, and gliosis can be observed. So how does grey matter damage arise? Several mechanisms have recently been proposed (figure 7), and either primary (arising within grey matter regions) or secondary (as a result of ongoing damage in the cerebral white matter) pathogenic processes could be involved.

\section{Secondary pathogenic mechanisms}

If grey matter pathology is to be thought of as largely secondary to inflammatory demyelinating damage in the white matter, several hypotheses can be devised. After demyelination in the white matter, changes in the expression of axonal sodium channels were observed in MS. ${ }^{122}$ More specifically, in active MS lesions, an abnormal, extensive, diffuse distribution of Nav1.2 and Nav1.6 sodium channels was found along long lengths of demyelinated axons that were positive for amyloid precursor protein (marker for axonal damage). Although this ion-channel redistribution is necessary to preserve at least some of the signal transduction properties of the injured axon, it requires a higher production of cellular ATP. With concurrent abnormalities found in mitochondrial functioning, ${ }^{123}$ for example, resulting from nitric-oxide exposure as part of the inflammatory cascade, ${ }^{124,125}$ demyelinated axons in the white matter have been proposed to enter a state of so-called "virtual hypoxia", 115 ultimately leading to neuroaxonal degeneration in the grey matter.

Evidence for alterations in the homoeostatis of the neurotransmitter glutamate was also found in MS, which could represent another secondary pathogenic mechanism leading to neuroaxonal degeneration in the grey matter. Demyelinated and injured axons in subcortical white matter, as well as oligodendrocytes, macroglia, and microglia/macrophages were found to express increased concentrations of ionotropic and metabotropic glutamate receptors and glutamate transporters compared with control brains, ${ }^{116-118}$ and treatment with ionotropic glutamate receptor antagonists was shown to improve clinical disability in experimental allergic encephalomyelitis (EAE). ${ }^{119,120}$ However, although glutamate dys-homoeostasis might indeed lead to axonal injury and to retrograde degeneration into the cortical grey matter, upregulated glutamate receptor subtypes and transporters might also function as a buffer, lowering extracellular glutamate concentrations, and thereby protecting the oligodendrocytes, which are known to be extremely vulnerable to glutamate excitotoxicity. ${ }^{120,121}$

Interestingly, gene transcripts of growth factor (ciliary neurotrophic factor) signalling pathways and the antiapoptotic molecule bcl2 were found to be upregulated in cortical neurons ${ }^{126}$ possibly reflecting a compensatory action in response to ongoing MS disease activity that might lead to axonal damage in the white matter and subsequent retrograde neuroaxonal degeneration. However, with regard to brain-derived neurotrophic factor, some controversy exists as to whether the Val66Met isoform of this growth factor leads to a more favourable 
disease course, because both reduction and preservation of grey matter volumes have been described in MS patients with this genetic background..$^{127,128}$ Neuroaxonal degeneration resulting from these secondary processes might in turn lead to loss of myelin in the grey matter, such that the lesions can be seen under the microscope in grey matter areas of MS brains.

\section{Primary pathogenic processes}

Although the above pathological processes might all seem important contributors to, or modulators of, grey matter damage in MS, increasing evidence also now points in the direction of the grey matter being primarily involved in the pathophysiology of MS. Of note, primary and secondary pathological processes are not mutually exclusive. Rather, they are likely to occur simultaneously in the MS brain, and to have a cumulative effect on demyelination and degeneration of axons in the grey matter. An example of how the MS cortex could be affected primarily (as opposed to secondarily to white matter pathological processes) was recently described by Magliozzi and co-workers, ${ }^{105}$ who suggested that meningeal inflammation (figure 5), rather than inflammation in the cortical lesions themselves, might cause cortical subpial demyelination as a side-effect. In this study, ectopic meningeal B-cell follicles in the leptomeninges of patients with MS were shown to be spatially related to cortical pathology. Because the pathological changes were seen to follow a gradient pattern in the superficial cortical layers in these cases, the authors hypothesised that a soluble cytotoxic/ myelinotoxic factor must be involved in the pathogenesis of cortical damage in MS. The exact nature of this myelinotoxic factor is not known. However, in EAE animals immunised with recombinant myelin oligodendrocyte protein, subpial cortical lesions much like those in MS were found to develop in association with band-like subpial microglia infiltration and immunoglobulin deposition on myelin sheaths. ${ }^{129,130}$ Whether an antibody-related pathological mechanism is also causally involved in the generation of demyelination of cortical axons in MS cannot be ruled out. Except for complement factor $\mathrm{C} 4 \mathrm{~d}$, which can be found associated with myelin in grey matter lesions in a small proportion of MS cases, no complement deposition could be observed in MS cortical lesions, in contrast to white matter lesions. ${ }^{31}$ Low concentrations of complement and possibly also of auto-antibodies might be present in the usually chronically affected cortical MS tissue, but they might remain below detection levels of the immunohistochemical techniques. However, antibodies can also induce demyelination of cortical axons in a complement-independent fashion through interaction with Fc-receptors on microglia and monocyte-derived macrophages or natural killer cells, although no signs of microglial activation or migration were seen in the grey matter lesions studied by Brink and colleagues. ${ }^{31}$

\section{Selective vulnerability of neurons and the "use-it-and- lose-it" principle}

Selective vulnerability of specific neuronal cell populations could be another reason for damage to grey matter regions in MS, similar to what is known for other neurodegenerative diseases, such as frontotemporal dementia (selective degeneration of Von Economo neurons). ${ }^{131}$ In rat EAE, a consistent decline in choline acetyltransferase activity was shown in the cerebral cortex, hippocampus, and basal forebrain, which was associated with learning and memory deficits. Treatment aimed at restoring acetylcholine concentrations through chronic administration of selective acetylcholinesterase inhibitors restored cognitive performance and choline acetyltransferase activity in these rats. ${ }^{132}$ Similar improvements in cognitive processing, including attention, memory, and executive functioning, were found for patients with MS on administration of the acetylcholinesterase inhibitor donepezil. ${ }^{133}$ In MS patients responding to a Stroop task, functional brain activation patterns were normalised through the action of the central acetylcholinesterase inhibitor rivastigmine.$^{87}$ Furthermore, a glutamate imbalance was shown to exist in the MS brain, leading to excitotoxicity and axonal damage. ${ }^{17,134}$ Future studies targeting the glutamatergic system by use of clinically tolerated glutamate-modulating agents such as memantine might therefore be considered in $\mathrm{MS}^{135}$ Finally, because sodium flux through sodium channels can trigger degeneration of axons that are already challenged in terms of propagation of their action potentials (as mentioned above), ${ }^{136}$ and different types of neurons express different repertoires of sodium channels that endow them with different firing patterns ${ }^{137}$ the differential tuning of various subpopulations of neurons, which depends on different expression patterns of sodium channels within them, might provide an additional basis for selective vulnerability.

Apart from the vulnerability of specific neurotransmitter systems, an interesting hypothesis in the field of ageing and Alzheimer's disease research postulates that cortical areas that are metabolically most highly and consistently active during life (eg, the default-mode network) might preferentially degenerate due to Alzheimer-related amyloid deposition. ${ }^{138}$ Whether a similar pattern of selective vulnerability of certain cortical areas also exists in MS (eg, the superior temporal gyrus and the superior and middle frontal gyri) is not known. ${ }^{34,40,41}$ Existence of this "use-it-and-lose-it" principle (as opposed to the more classically cited "use-it-or-lose-it" concept) should be investigated in future studies.

\section{Conclusions and future directions}

Over the past few years, it has become clear that grey matter damage in MS is extensive, it is already present in early disease stages, it increases with disease duration, and it is clinically relevant. More research is warranted to clarify the spatiotemporal relations between white matter and grey matter damage. In light of this research, the 


\section{Search strategy and selection criteria}

References for this Review were identified through PubMed searches from 1900 to April, 2008, by use of the search terms "multiple sclerosis", "grey/gray matter", "cortical", and/or "neurodegeneration", in combination with topic-specific terms such as "mitochondrial", "glutamate", "atrophy", "functional MRI", "positron emission tomography", "EAE", "antibody", and "memantine". Articles were also identified through searches of the authors' own files, and recent, important developments in the area of grey matter research in MS presented at the 23rd European Congress for Treatment and Research in Multiple Sclerosis (ECTRIMS), Prague, Czech Republic (Oct 11-14, 2007), were discussed in the Review if appropriate. Only papers published in the English language were reviewed.

innovative combination of structural and functional connectivity measures might be expected to generate important insights into the effect of (focal) damage to white matter tracts on (regional) cortical integrity.

At present, we are unsure of the causes of demyelination and degeneration of neuroaxonal elements in grey matter regions in MS. Disease mechanisms leading to grey matter damage might be different from those leading to white matter damage in MS, but this is still a topic of debate. Meningeal inflammation as a possible cause of subpial cortical demyelination is one of several exciting new hypotheses currently being explored. Also, regional cortical thickness studies have shown that atrophy is predominantly found in frontal and temporal cortical areas. This finding tentatively opens doors to new ideas on selective vulnerability of neuronal subpopulations in MS, as was already shown for other neurodegenerative diseases. Whether and how much the different pathomechanisms reviewed here have a significant contribution in demyelination of cortical grey matter (and possible neuroaxonal degeneration), and whether demyelination in different grey matter areas, such as the neocortex and cerebellar cortex, spinal cord, basal ganglia, and hippocampus, might arise from similar causal pathways, remains to be elucidated.

An increase in knowledge regarding damage to grey matter structures in MS might also result in a more targeted pharmacotherapeutic approach to the disease, with focus on cognitive decline and specific neurotransmitter systems (ie, acetylcholine), for example. For the coming years, the combination of advanced neuroimaging, clinico-cognitive, neuropathological, immunological, and genetic expression studies seems to be the strategy of choice in disentangling MS grey matter pathology, which at present remains enigmatic.

\section{Contributors}

Both authors were involved in designing, writing, and editing the manuscript. Both authors have seen and agree with the contents of the final version of the paper. The authors disclosed no competing financial interests.

\section{Conflicts of interest}

We have no conflicts of interest.

\section{Acknowledgments}

The MS Centre Amsterdam and JJGG were sponsored by the Dutch MS Research Foundation (grant 05-358c). The authors wish to thank L Bö (Department of Neurology, Haukeland University Hospital, Bergen, Norway) for providing figure 1, H Lassmann (Centre for Brain Research, Medical University of Vienna, Vienna, Austria) for providing figure 3, E-J Kooi (Department of Neuropathology, VU University Medical Centre, Amsterdam, Netherlands) for providing figure 6, and H Vrenken and S D Roosendaal for carefully reading the manuscript.

\section{References}

1 Sander M. Hirnrindenbefunde bei multipler Sklerose. Monatschrift Psychiatrie Neurol 1898; IV: 427-36.

2 Dinkler M. Zur Kasuistik der multiplen Herdsklerose des Gehirns und Ruckenmarks. Deuts Zeits $f$ Nervenheilk 1904; 26: 233-47.

3 Schob F. Ein Beitrag zur patologischen Anatomie der multiplen Sklerose. Monatschrift Psychiatrie Neurol 1907; 22: 62-87.

4 Dawson JW. The histology of multiple sclerosis. Trans $R$ Soc (Edinb) 1916; 50: 517-740.

5 Brownell B, Hughes JT. The distribution of plaques in the cerebrum in multiple sclerosis. J Neurol Neurosurg Psychiatry 1962; 25: 315-20.

6 Bö L, Geurts JJ, van der Valk P, Polman C, Barkhof F. Lack of correlation between cortical demyelination and white matter pathologic changes in multiple sclerosis. Arch Neurol 2007; 64: 76-80.

7 Bö L, Vedeler CA, Nyland HI, Trapp BD, Mörk SJ. Subpial demyelination in the cerebral cortex of multiple sclerosis patients. J Neuropathol Exp Neurol 2003; 62: 723-32.

8 Bö L, Vedeler CA, Nyland H, Trapp BD, Mörk SJ. Intracortical multiple sclerosis lesions are not associated with increased lymphocyte infiltration. Mult Scler 2003; 9: 323-31.

9 Peterson JW, Bö L, Mörk S, Chang A, Trapp BD. Transected neurites, apoptotic neurons, and reduced inflammation in cortical multiple sclerosis lesions. Ann Neurol 2001; 50: 389-400.

10 Kutzelnigg A, Lucchinetti CF, Stadelmann C, et al. Cortical demyelination and diffuse white matter injury in multiple sclerosis. Brain 2005; 128: 2705-12.

11 Barkhof F. The clinico-radiological paradox in multiple sclerosis revisited. Curr Opin Neurol 2002; 15: 239-45.

12 Moriarty DM, Blackshaw AJ, Talbot PR, et al. Memory dysfunction in multiple sclerosis corresponds to juxtacortical lesion load on fast fluid-attenuated inversion-recovery MR images. AJNR Am J Neuroradiol 1999; 20: 1956-62.

13 Lazeron RH, Langdon DW, Filippi M, et al. Neuropsychological impairment in multiple sclerosis patients: the role of (juxta)cortical lesion on FLAIR. Mult Scler 2000; 6: 280-85.

14 Rovaris M, Filippi M, Minicucci L, et al. Cortical/subcortical disease burden and cognitive impairment in patients with multiple sclerosis. AJNR Am J Neuroradiol 2000; 21: 402-08.

15 Sokic DV, Stojsavljevic N, Drulovic J, et al. Seizures in multiple sclerosis. Epilepsia 2001; 42: 72-79.

16 Spatt J, Chaix R, Mamoli B. Epileptic and non-epileptic seizures in multiple sclerosis. J Neurol 2001; 248: 2-9.

17 Benedict RH, Weinstock-Guttman B, Fishman I, et al. Prediction of neuropsychological impairment in multiple sclerosis: comparison of conventional magnetic resonance imaging measures of atrophy and lesion burden. Arch Neurol 2004; 61: 226-30.

18 Feinstein A, Roy P, Lobaugh N, et al. Structural brain abnormalities in multiple sclerosis patients with major depression. Neurology 2004; 62: 586-90.

19 Rao SM, Leo GJ, Bernardin L, Unverzagt F. Cognitive dysfunction in multiple sclerosis. I. Frequency, patterns, and prediction. Neurology 1991; 41: 685-91.

20 Rao SM. Neuropsychology of multiple sclerosis. Curr Opin Neurol 1995; 8: 216-20.

21 Amato MP, Bartolozzi ML, Zipoli V, et al. Neocortical volume decrease in relapsing-remitting MS patients with mild cognitive impairment. Neurology 2004; 63: 89-93.

22 Amato MP, Portaccio E, Goretti B, et al. Association of neocortical volume changes with cognitive deterioration in relapsing-remitting multiple sclerosis. Arch Neurol 2007; 64: 1157-61. 
23 Zarei M, Chandran S, Compston A, Hodges J. Cognitive presentation of multiple sclerosis: evidence for a cortical variant. J Neurol Neurosurg Psychiatry 2003; 74: 872-77.

24 Zarei M. Clinical characteristics of cortical multiple sclerosis. J Neurol Sci 2006; 245: 53-58

25 Geurts JJ, Bö L, Roosendaal SD, et al. Extensive hippocampal demyelination in multiple sclerosis. J Neuropathol Exp Neurol 2007; 66: 819-27.

26 Vercellino M, Plano F, Votta B, et al. Grey matter pathology in multiple sclerosis. J Neuropathol Exp Neurol 2005; 64: 1101-07.

27 Huitinga I, De Groot CJ, van der Valk P, et al. Hypothalamic lesions in multiple sclerosis. J Neuropathol Exp Neurol 2001; 60: 1208-18.

28 Gilmore CP, Bö L, Owens T, et al. Spinal cord gray matter demyelination in multiple sclerosis-a novel pattern of residual plaque morphology. Brain Pathol 2006; 16: 202-08.

29 Calabrese M, De Stefano N, Atzori M, et al. Detection of cortical inflammatory lesions by double inversion recovery magnetic resonance imaging in patients with multiple sclerosis. Arch Neurol 2007; 64: 1416-22.

30 Huitinga I, Erkut ZA, van Beurden D, Swaab DF. Impaired hypothalamus-pituitary-adrenal axis activity and more severe multiple sclerosis with hypothalamic lesions. Ann Neurol 2004; 55: 37-45.

31 Brink BP, Veerhuis R, Breij EC, et al. The pathology of multiple sclerosis is location-dependent: no significant complement activation is detected in purely cortical lesions. J Neuropathol Exp Neurol 2005; 64: 147-55.

32 van Horssen J, Brink BP, de Vries HE, van der Valk P, Bö L. The blood-brain barrier in cortical multiple sclerosis lesions. J Neuropathol Exp Neurol 2007; 66: 321-28.

33 Wegner C, Esiri MM, Chance SA, Palace J, Matthews PM. Neocortical neuronal, synaptic, and glial loss in multiple sclerosis. Neurology 2006; 67: 960-67.

34 Sailer M, Fischl B, Salat D, et al. Focal thinning of the cerebral cortex in multiple sclerosis. Brain 2003; 126: 1734-44.

35 De Stefano N, Matthews PM, Filippi M, et al. Evidence of early cortical atrophy in MS: relevance to white matter changes and disability. Neurology 2003; 60: 1157-62.

36 Chen JT, Narayanan S, Collins DL, et al. Relating neocortical pathology to disability progression in multiple sclerosis using MRI. Neuroimage 2004; 23: 1168-75.

37 Prinster A, Quarantelli M, Orefice G, et al. Grey matter loss in relapsing-remitting multiple sclerosis: a voxel-based morphometry study. Neuroimage 2006; 29: 859-67.

38 Sepulcre J, Sastre-Garriga J, Cercignani M, et al. Regional gray matter atrophy in early primary progressive multiple sclerosis a voxel-based morphometry study. Arch Neurol 2006; 63: 1175-80.

39 Audoin B, Davies GR, Finisku L, et al. Localization of grey matter atrophy in early RRMS: a longitudinal study. J Neurol 2006; 253: $1495-501$.

40 Lumsden CE. The neuropathology of multiple sclerosis. In: Vinken PJ, Bruyn GW, eds. Handbook of clinical neurology, vol. 9. Multiple sclerosis and other demyelinating diseases. Amsterdam: Elsevier, 1970; 217-309.

41 Wegner C, Matthews PM. A new view of the cortex, new insights into multiple sclerosis. Brain 2003; 126: 1719-21.

42 Vrenken H, Geurts JJ, Knol DL, et al. Whole-brain T1 mapping in multiple sclerosis: global changes of normal-appearing gray and white matter. Radiology 2006; 240: 811-20.

43 Charil A, Dagher A, Lerch JP, et al. Focal cortical atrophy in multiple sclerosis: relation to lesion load and disability. Neuroimage 2007; 34: 509-17.

44 Geurts JJ, Bö L, Pouwels PJ, et al. Cortical lesions in multiple sclerosis: combined postmortem MR imaging and histopathology. AJNR Am J Neuroradiol 2005; 26: 572-77.

45 Geurts JJ, Blezer EL, Vrenken H, et al. Does high-field MR imaging improve cortical lesion detection in multiple sclerosis? J Neurol 2008; 255: 183-91.

46 Geurts JJ, Pouwels PJ, Uitdehaag BM, et al. Intracortical lesions in multiple sclerosis: improved detection with 3D double inversionrecovery MR imaging. Radiology 2005; 236: 254-60.

47 Pouwels PJ, Kuijer JP, Mugler JP III, Guttmann CR, Barkhof F. Human gray matter: feasibility of single-slab 3D double inversionrecovery high-spatial-resolution MR imaging. Radiology 2006; 241: 873-79.
48 Bagnato F, Butman JA, Gupta S, et al. In vivo detection of cortical plaques by MR imaging in patients with multiple sclerosis. AJNR Am J Neuroradiol 2006; 27: 2161-67.

49 Cercignani M, Bozzali M, Iannucci G, Comi G, Filippi M. Magnetisation transfer ratio and mean diffusivity of norma appearing white and grey matter from patients with multiple sclerosis. J Neurol Neurosurg Psychiatry 2001; 70: 311-17.

50 Davies GR, Ramio-Torrenta L, Hadjiprocopis A, et al. Evidence for grey matter MTR abnormality in minimally disabled patients with early relapsing-remitting multiple sclerosis. J Neurol Neurosurg Psychiatry 2004; 75: 998-1002.

51 Ramio-Torrenta L, Sastre-Garriga J, Ingle GT, et al. Abnormalities in normal appearing tissues in early primary progressive multiple sclerosis and their relation to disability: a tissue specific magnetisation transfer study. J Neurol Neurosurg Psychiatry 2006; 77: 40-45.

52 Manfredonia F, Ciccarelli O, Khaleeli Z, et al. Normal-appearing brain $\mathrm{T} 1$ relaxation time predicts disability in early primary progressive multiple sclerosis. Arch Neurol 2007; 64: 411-15.

53 Rovaris M, Judica E, Gallo A, et al. Grey matter damage predicts the evolution of primary progressive multiple sclerosis at 5 years. Brain 2006; 129: 2628-34.

54 Vrenken H, Pouwels PJ, Geurts JI, et al. Altered diffusion tensor in multiple sclerosis normal-appearing brain tissue: cortical diffusion changes seem related to clinical deterioration. J Magn Reson Imaging 2006; 23: 628-36.

55 Chard DT, Griffin CM, McLean MA, et al. Brain metabolite changes in cortical grey and normal-appearing white matter in clinically early relapsing-remitting multiple sclerosis. Brain 2002; 125: 2342-52.

56 Geurts JJ, Reuling IE, Vrenken H, et al. MR spectroscopic evidence for thalamic and hippocampal, but not cortical, damage in multiple sclerosis. Magn Reson Med 2006; 55: 478-83.

57 Kapeller P, McLean MA, Griffin CM, et al. Preliminary evidence for neuronal damage in cortical grey matter and normal appearing white matter in short duration relapsing-remitting multiple sclerosis: a quantitative MR spectroscopic imaging study. J Neurol 2001; 248: 131-38.

58 Vrenken H, Geurts JJ. Gray and normal-appearing white matter in multiple sclerosis: an MRI perspective. Expert Rev Neurother 2007; 7: 271-79.

59 Miller DH, Thompson AJ, Filippi M. Magnetic resonance studies of abnormalities in the normal appearing white matter and grey matter in multiple sclerosis. J Neurol 2003; 250: 1407-19.

60 Benedict RH, Zivadinov R, Carone DA, et al. Regional lobar atrophy predicts memory impairment in multiple sclerosis. AJNR Am J Neuroradiol 2005; 26: 1824-31.

61 Fisher E, Rudick RA, Cutter G, et al. Relationship between brain atrophy and disability: an 8-year follow-up study of multiple sclerosis patients. Mult Scler 2000; 6: 373-77.

62 Tedeschi G, Lavorgna L, Russo P, et al. Brain atrophy and lesion load in a large population of patients with multiple sclerosis. Neurology 2005; 65: 280-85.

63 Agosta F, Rovaris M, Pagani E, et al. Magnetization transfer MRI metrics predict the accumulation of disability 8 years later in patients with multiple sclerosis. Brain 2006; 129: 2620-27.

64 Oreja-Guevara C, Charil A, Caputo D, et al. Magnetization transfe magnetic resonance imaging and clinical changes in patients with relapsing-remitting multiple sclerosis. Arch Neurol 2006; 63: 736-40.

65 Khaleeli Z, Cercignani M, Audoin B, et al. Localized grey matter damage in early primary progressive multiple sclerosis contributes to disability. Neuroimage 2007; 37: 253-61.

66 Dehmeshki J, Chard DT, Leary SM, et al. The normal appearing grey matter in primary progressive multiple sclerosis: a magnetisation transfer imaging study. J Neurol 2003; 250: 67-74.

67 Van Au DM, Audoin B, Le Fur Y, et al. Relationships between gray matter metabolic abnormalities and white matter inflammation in patients at the very early stage of MS: a MRSI study. J Neurol 2007; 254: 914-23.

68 Pulizzi A, Rovaris M, Judica E, et al. Determinants of disability in multiple sclerosis at various disease stages: a multiparametric magnetic resonance study. Arch Neurol 2007; 64: 1163-68.

69 Sastre-Garriga J, Ingle GT, Chard DT, et al. Metabolite changes in normal-appearing gray and white matter are linked with disability in early primary progressive multiple sclerosis. Arch Neurol 2005 62: 569-73. 
70 Cifelli A, Arridge M, Jezzard P, et al. Thalamic neurodegeneration in multiple sclerosis. Ann Neurol 2002; 52: 650-53.

71 Bakshi R, Benedict RH, Bermel RA, et al. T2 hypointensity in the deep gray matter of patients with multiple sclerosis: a quantitative magnetic resonance imaging study. Arch Neurol 2002; 59: 62-68

72 Caramanos Z, Narayanan S, Arnold DL. ${ }^{1}$ H-MRS quantification of tNA and tCr in patients with multiple sclerosis: a meta-analytic review. Brain 2005; 128: 2483-506.

73 Geurts JJ, Barkhof F, Castelijns JA, et al. Quantitative ${ }^{1} \mathrm{H}$-MRS of healthy human cortex, hippocampus, and thalamus: metabolite concentrations, quantification precision, and reproducibility. J Magn Reson Imaging 2004; 20: 366-71.

74 Bot JC, Barkhof F, Polman CH, et al. Spinal cord abnormalities in recently diagnosed MS patients: added value of spinal MRI examination. Neurology 2004; 62: 226-33.

75 Barkhof F, Filippi M, Miller DH, et al. Comparison of MRI criteria at first presentation to predict conversion to clinically definite multiple sclerosis. Brain 1997; 120: 2059-69.

76 Polman CH, Reingold SC, Edan G, et al. Diagnostic criteria for multiple sclerosis: 2005 revisions to the "McDonald Criteria". Ann Neurol 2005; 58: 840-46.

77 Nijeholt GJ, Barkhof F, Scheltens P, et al. MR of the spinal cord in multiple sclerosis: relation to clinical subtype and disability. AJNR Am J Neuroradiol 1997; 18: 1041-48.

78 Filippi M, Bozzali M, Horsfield MA, et al. A conventional and magnetization transfer MRI study of the cervical cord in patients with MS. Neurology 2000; 54: 207-13.

79 Agosta F, Absinta M, Sormani MP, et al. In vivo assessment of cervical cord damage in MS patients: a longitudinal diffusion tensor MRI study. Brain 2007; 130: 2211-19.

80 Agosta F, Pagani E, Caputo D, Filippi M. Associations between cervical cord gray matter damage and disability in patients with multiple sclerosis. Arch Neurol 2007; 64: 1302-05.

81 Ciccarelli O, Wheeler-Kingshott CA, McLean MA, et al. Spinal cord spectroscopy and diffusion-based tractography to assess acute disability in multiple sclerosis. Brain 2007; 130: 2220-31.

82 Agosta F, Valsasina P, Caputo D, Stroman PW, Filippi M. Tactileassociated recruitment of the cervical cord is altered in patients with multiple sclerosis. Neuroimage 2008; 39: 1542-48.

83 Lee M, Reddy H, Johansen-Berg H, et al. The motor cortex shows adaptive functional changes to brain injury from multiple sclerosis. Ann Neurol 2000; 47: 606-13.

84 Reddy H, Narayanan S, Arnoutelis R, et al. Evidence for adaptive functional changes in the cerebral cortex with axonal injury from multiple sclerosis. Brain 2000; 123: 2314-20.

85 Filippi M, Rocca MA, Falini A, et al. Correlations between structural CNS damage and functional MRI changes in primary progressive MS. Neuroimage 2002; 15: 537-46.

86 Rocca MA, Pagani E, Ghezzi A, et al. Functional cortical changes in patients with multiple sclerosis and nonspecific findings on conventional magnetic resonance imaging scans of the brain Neuroimage 2003; 19: 826-36.

87 Parry AM, Scott RB, Palace J, Smith S, Matthews PM. Potentially adaptive functional changes in cognitive processing for patients with multiple sclerosis and their acute modulation by rivastigmine. Brain 2003; 126: 2750-60

88 Cader S, Cifelli A, Abu-Omar Y, Palace J, Matthews PM. Reduced brain functional reserve and altered functional connectivity in patients with multiple sclerosis. Brain 2006; 129: 527-37.

89 Wegner C, Filippi M, Korteweg T, et al. Relating functional changes during hand movement to clinical parameters in patients with multiple sclerosis in a multi-centre fMRI study. Eur J Neurol 2008 15: 113-22.

90 Audoin B, Ibarrola D, Ranjeva JP, et al. Compensatory cortical activation observed by fMRI during a cognitive task at the earliest stage of MS. Hum Brain Mapp 2003; 20: 51-58.

91 Rocca MA, Colombo B, Falini A, et al. Cortical adaptation in patients with MS: a cross-sectional functional MRI study of disease phenotypes. Lancet Neurol 2005; 4: 618-26.

92 Rocca MA, Pagani E, Absinta M, et al. Altered functional and structural connectivities in patients with MS: a 3-T study. Neurology 2007; 69: 2136-45.
93 Ciccarelli O, Toosy AT, Marsden JF, et al. Functional response to active and passive ankle movements with clinical correlations in patients with primary progressive multiple sclerosis. J Neurol 2006; 253: 882-91.

94 Audoin B, Guye M, Reuter F, et al. Structure of WM bundles constituting the working memory system in early multiple sclerosis: a quantitative DTI tractography study. Neuroimage 2007; 36: $1324-30$.

95 Smith SM, Jenkinson M, Johansen-Berg H, et al. Tract-based spatial statistics: voxelwise analysis of multi-subject diffusion data. Neuroimage 2006; 31: 1487-505.

96 Ge Y, Law M, Johnson G, et al. Preferential occult injury of corpus callosum in multiple sclerosis measured by diffusion tensor imaging. J Magn Reson Imaging 2004; 20: 1-7.

97 Narayanan S, Francis SJ, Sled JG, et al. Axonal injury in the cerebra normal-appearing white matter of patients with multiple sclerosis is related to concurrent demyelination in lesions but not to concurren demyelination in normal-appearing white matter. Neuroimage 2006; 29: 637-42.

98 Derache N, Marie RM, Constans JM, Defer GL. Reduced thalamic and cerebellar rest metabolism in relapsing-remitting multiple sclerosis, a positron emission tomography study: correlations to lesion load. J Neurol Sci 2006; 245: 103-09.

99 Paulesu E, Perani D, Fazio F, et al. Functional basis of memory impairment in multiple sclerosis: a $\left[{ }^{18}\right.$ F]FDG PET study. Neuroimage 1996; 4: 87-96.

100 Blinkenberg M, Rune K, Jensen CV, et al. Cortical cerebral metabolism correlates with MRI lesion load and cognitive dysfunction in MS. Neurology 2000; 54: 558-64.

101 Banati RB, Newcombe J, Gunn RN, et al. The peripheral benzodiazepine binding site in the brain in multiple sclerosis: quantitative in vivo imaging of microglia as a measure of disease activity. Brain 2000; 123: 2321-37.

102 Inglese M, Park SJ, Johnson G, et al. Deep gray matter perfusion in multiple sclerosis: dynamic susceptibility contrast perfusion magnetic resonance imaging at 3 T. Arch Neurol 2007; 64: 196-202.

103 Rashid W, Parkes LM, Ingle GT, et al. Abnormalities of cerebral perfusion in multiple sclerosis. J Neurol Neurosurg Psychiatry 2004; 75: 1288-93.

104 Inglese M, Adhya S, Johnson G, et al. Perfusion magnetic resonance imaging correlates of neuropsychological impairment in multiple sclerosis. J Cereb Blood Flow Metab 2008; 28: 164-71.

105 Magliozzi R, Howell O, Vora A, et al. Meningeal B-cell follicles in secondary progressive multiple sclerosis associate with early onset of disease and severe cortical pathology. Brain 2007; 130: 1089-104.

106 Serafini B, Rosicarelli B, Franciotta D, et al. Dysregulated EpsteinBarr virus infection in the multiple sclerosis brain. J Exp Med 2007; 204: 2899-912.

107 Kutzelnigg A, Faber-Rod JC, Bauer J, et al. Widespread demyelination in the cerebellar cortex in multiple sclerosis. Brain Pathol 2007; 17: 38-44.

108 Bozzali M, Cercignani M, Sormani MP, Comi G, Filippi M. Quantification of brain gray matter damage in different MS phenotypes by use of diffusion tensor MR imaging. AJNR Am J Neuroradiol 2002; 23: 985-88.

109 Ge Y, Grossman RI, Udupa JK, et al. Magnetization transfer ratio histogram analysis of normal-appearing gray matter and normal-appearing white matter in multiple sclerosis. J Comput Assist Tomogr 2002; 26: 62-68.

110 Oreja-Guevara C, Rovaris M, Iannucci G, et al. Progressive gray matter damage in patients with relapsing-remitting multiple sclerosis: a longitudinal diffusion tensor magnetic resonance imaging study. Arch Neurol 2005; 62: 578-84.

111 Sastre-Garriga J, Ingle GT, Chard DT, et al. Grey and white matte volume changes in early primary progressive multiple sclerosis: a longitudinal study. Brain 2005; 128: 1454-60.

112 Audoin B, Davies G, Rashid W, et al. Voxel-based analysis of grey matter magnetization transfer ratio maps in early relapsing remitting multiple sclerosis. Mult Scler 2007; 13: 483-89.

113 Bruck W. Cortical pathology in MS: is it really a late phenomenon? Mult Scler 2007; 13: S12.

114 Damoiseaux JS, Rombouts SA, Barkhof F, et al. Consistent restingstate networks across healthy subjects. Proc Natl Acad Sci USA 2006; 103: 13848-53. 
115 Stys PK. Axonal degeneration in multiple sclerosis: is it time for neuroprotective strategies? Ann Neurol 2004; 55: 601-03.

116 Geurts JJ, Wolswijk G, Bö L, et al. Expression patterns of group III metabotropic glutamate receptors mGluR4 and mGluR8 in multiple sclerosis lesions. J Neuroimmunol 2005; 158: 182-90.

117 Geurts JJ, Wolswijk G, Bö L, et al. Altered expression patterns of group I and II metabotropic glutamate receptors in multiple sclerosis. Brain 2003; 126: 1755-66.

118 Newcombe J, Uddin A, Dove R, et al. Glutamate receptor expression in multiple sclerosis lesions. Brain Pathol 2008; 18: 52-61.

119 Pitt D, Werner P, Raine CS. Glutamate excitotoxicity in a model of multiple sclerosis. Nat Med 2000; 6: 67-70.

120 Werner P, Pitt D, Raine CS. Glutamate excitotoxicity-a mechanism for axonal damage and oligodendrocyte death in Multiple Sclerosis? J Neural Transm Suppl 2000; 60: 375-85.

121 Domercq M, Etxebarria E, Perez-Samartin A, Matute C. Excitotoxic oligodendrocyte death and axonal damage induced by glutamate transporter inhibition. Glia 2005; 52: 36-46.

122 Craner MJ, Newcombe J, Black JA, et al. Molecular changes in neurons in multiple sclerosis: altered axonal expression of Nav1.2 and Nav1.6 sodium channels and $\mathrm{Na}+\mathrm{Ca} 2+$ exchanger. Proc Natl Acad Sci USA 2004; 101: 8168-73.

123 Dutta R, McDonough J, Yin X, et al. Mitochondrial dysfunction as a cause of axonal degeneration in multiple sclerosis patients. Ann Neurol 2006; 59: 478-89.

124 Bö L, Dawson TM, Wesselingh S, et al. Induction of nitric oxide synthase in demyelinating regions of multiple sclerosis brains. Ann Neurol 1994; 36: 778-86.

125 Kapoor R, Davies M, Blaker PA, Hall SM, Smith KJ. Blockers of sodium and calcium entry protect axons from nitric oxide-mediated degeneration. Ann Neurol 2003; 53: 174-80.

126 Dutta R, McDonough J, Chang A, et al. Activation of the ciliary neurotrophic factor (CNTF) signalling pathway in cortical neurons of multiple sclerosis patients. Brain 2007; 130: 2566-76.

127 Liguori M, Fera F, Gioia MC, et al. Investigating the role of brainderived neurotrophic factor in relapsing-remitting multiple sclerosis. Genes Brain Behav 2007; 6: 177-83.

128 Zivadinov R, Weinstock-Guttman B, Benedict R, et al. Preservation of gray matter volume in multiple sclerosis patients with the Met allele of the rs6265 (Val66Met) SNP of brain-derived neurotrophic factor. Hum Mol Genet 2007; 16: 2659-68.
129 Merkler D, Schmelting B, Czeh B, et al. Myelin oligodendrocyte glycoprotein-induced experimental autoimmune encephalomyelitis in the common marmoset reflects the immunopathology of pattern II multiple sclerosis lesions. Mult Scler 2006; 12: 369-74.

130 Storch MK, Bauer J, Linington C, et al. Cortical demyelination can be modeled in specific rat models of autoimmune encephalomyelitis and is major histocompatability complex (MHC) haplotype-related. J Neuropathol Exp Neurol 2006; 65: 1137-42.

131 Seeley WW, Carlin DA, Allman JM, et al. Early frontotemporal dementia targets neurons unique to apes and humans. Ann Neurol 2006; 60: 660-67.

132 D'Intino G, Paradisi M, Fernandez M, et al. Cognitive deficit associated with cholinergic and nerve growth factor downregulation in experimental allergic encephalomyelitis in rats. Proc Natl Acad Sci USA 2005; 102: 3070-75.

133 Greene YM, Tariot PN, Wishart H, et al. A 12-week, open trial of donepezil hydrochloride in patients with multiple sclerosis and associated cognitive impairments. J Clin Psychopharmacol 2000; 20: $350-56$.

134 Werner P, Pitt D, Raine CS. Multiple sclerosis: altered glutamate homeostasis in lesions correlates with oligodendrocyte and axonal damage. Ann Neurol 2001; 50: 169-80.

135 Stys PK, Lipton SA. White matter NMDA receptors: an unexpected new therapeutic target? Trends Pharmacol Sci 2007; 28: 561-66.

136 Waxman SG, Black JA, Ransom BR, Stys PK. Anoxic injury of rat optic nerve: ultrastructural evidence for coupling between $\mathrm{Na}^{+}$ influx and $\mathrm{Ca}(2+)$-mediated injury in myelinated CNS axons. Brain Res 1994; 644: 197-204.

137 Waxman SG. The neuron as a dynamic electrogenic machine: modulation of sodium-channel expression as a basis for functional plasticity in neurons. Philos Trans R Soc Lond B Biol Sci 2000; 355: 199-213.

138 Buckner RL, Snyder AZ, Shannon BJ, et al. Molecular, structural, and functional characterization of Alzheimer's disease: evidence for a relationship between default activity, amyloid, and memory. J Neurosci 2005; 25: 7709-17. 